WellBeing International

WBI Studies Repository

2010

\title{
The Current Scientific and Legal Status of Alternative Methods to the LD50 Test for Botulinum Neurotoxin Potency Testing
}

\author{
Sarah Adler \\ Centre for Documentation and Evaluation of Alternatives to Animal Experiments (ZEBET) \\ Gerd Bicker \\ University of Veterinary Medicine Hannover \\ Hans Bigalke \\ Hannover Medical School \\ Christopher Bishop \\ Wickham Laboratories \\ Jörg Blümel \\ Merz Pharmaceuticals
}

See next page for additional authors

Follow this and additional works at: https://www.wellbeingintlstudiesrepository.org/acwp_arte

Part of the Bioethics and Medical Ethics Commons, Laboratory and Basic Science Research

Commons, and the Research Methods in Life Sciences Commons

\section{Recommended Citation}

Adler, S., Bicker, G., Bigalke, H., Bishop, C., Blümel, J., Dressler, D., ... \& Liebsch, M. (2010, August). The current scientific and legal status of alternative methods to the LD50 test for botulinum neurotoxin potency testing. In The report and recommendations of a ZEBET Expert Meeting. ATLA (Vol. 38, No. 4, pp. 315-330).

This material is brought to you for free and open access by WellBeing International. It has been accepted for inclusion by an authorized administrator of the WBI Studies Repository. For more information, please contact wbisr-info@wellbeingintl.org.

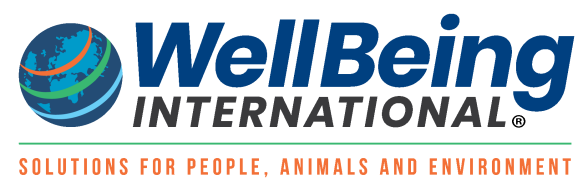




\section{Authors}

Sarah Adler, Gerd Bicker, Hans Bigalke, Christopher Bishop, Jörg Blümel, Dirk Dressler, Joan Fitzgerald, Frank Gessler, Heide Heuschen, Birgit Kegel, Andreas Luch, Catherine Milne, Andrew Pickett, Heidemarie Ratsch, Irmela Ruhdel, Dorothea Sesardic, Martin Stephens, Gerhard Stiens, Peter D. Thornton, René Thürmer, Martin Vey, Horst Spielmann, Barbara Grune, and Manfred Liebsch 


\title{
The Current Scientific and Legal Status of Alternative Methods to the LD50 Test for Botulinum Neurotoxin Potency Testing
}

\author{
The Report and Recommendations of a ZEBET Expert Meeting
}

\begin{abstract}
Sarah Adler, ${ }^{1}$ Gerd Bicker, ${ }^{2}$ Hans Bigalke, ${ }^{3}$ Christopher Bishop, ${ }^{4}$ Jörg Blümel, ${ }^{5}$ Dirk Dressler, ${ }^{6}$ Joan Fitzgerald,7 Frank Gessler, 8 Heide Heuschen, ${ }^{9}$ Birgit Kegel,10 Andreas Luch, 1,11 Catherine Milne, 12 Andrew Pickett, 13 Heidemarie Ratsch,14 Irmela Ruhdel,15 Dorothea Sesardic, 16 Martin Stephens, 17 Gerhard Stiens, 18 Peter D. Thornton, ${ }^{19}$ René Thürmer, ${ }^{9}$ Martin Vey, ${ }^{5}$ Horst Spielmann, 1,20 Barbara Grune ${ }^{1}$ and Manfred Liebsch 1
\end{abstract}

${ }^{1}$ Centre for Documentation and Evaluation of Alternatives to Animal Experiments (ZEBET), Federal Institute for Risk Assessment (BfR), Berlin, Germany; 2University of Veterinary Medicine Hannover, Division of Cell Biology, Hannover, Germany; 3Institute of Toxicology, Medical School of Hannover, Hannover, Germany; 4Wickham Laboratories, Wickham, Hampshire, UK; 5Merz Pharmaceuticals GmbH, Frankfurt (Main), Germany; ${ }^{6}$ Department of Neurology, Rostock University, Germany; 7Allergan, Inc., Irvine, CA, USA; 8Institute for Applied Biotechnology in the Tropics at the University of Göttingen, Göttingen, Germany; ${ }^{9}$ Federal Institute for Drugs and Medical Devices (BfArM), Bonn, Germany; 10Paul-Ehrlich-Institut, Federal Institute for Vaccines and Biomedicines, Langen, Germany; ${ }^{11}$ Department of Product Safety, Federal Institute for Risk Assessment, Berlin, Germany; ${ }^{12}$ European Directorate of the Quality of Medicines and Health Care (EDQM), Strasbourg, France; 13/psen Biopharm Limited, Wrexham, UK; ${ }^{14}$ Landesamt für Gesundheit und Soziales Berlin (LAGeSo), Berlin, Germany; 15Akademie für Tierschutz, Neubiberg, Germany; 16National Institute for Biological Standards and Control (NIBSC), Health Protection Agency (HPA), Potters Bar, Hertfordshire, UK; 17The Humane Society of the United States (HSUS), Washington, USA; 18Federal Ministry of Food, Agriculture and Consumer Protection (BMELV), Bonn, Germany; 19Home Office, ASPI, London, UK; 20Free University Berlin, Berlin, Germany

\section{Preface}

An Expert Meeting on The Current Scientific and Legal Status of Alternative Methods to the LD50 Test for Botulinum Neurotoxin (BoNT) Potency Testing was organised by the Centre for Documentation and Evaluation of Alternatives to Animal Experiments (ZEBET) at the Federal Institute for Risk Assessment (BfR), and took place on 27-28 April 2009, in Berlin, Germany. The goal of ZEBET is to promote primarily the replacement of legally required animal experiments with valid alternative test methods, to reduce the numbers of test animals used to the absolutely necessary level, and to alleviate their pain and suffering. ZEBET's tasks are the documentation, assessment and promotion of alternative methods to animal experiments on a national and international level, and the initiation of research and development, including the funding of smaller research projects.
The Expert Meeting on alternative methods for Botulinum neurotoxin (BoNT) potency testing was held at the BfR as the result of a decree from the German Federal Ministry of Food, Agriculture and Consumer Protection (BMELV), after it was informed by German state authorities about an increase in animal numbers being used in the LD50 potency test in mice for the purposes of BoNT potency testing. Ethical concerns have been raised in both Europe and the United States about the animal suffering involved in this type of testing, especially in the context of BoNT's aesthetic applications. There had been increased public pressure on the German Government, due to successful campaigning by animal welfare/anti-vivisectionist organisations on the issue of BoNT animal testing. As a result, the BMELV asked ZEBET to assess the status of the different alternative methods to the BoNT LD50 potency test that are cited in the European Pharmacopoeia (EP) 6.0, Monograph 2113, Botulinum Toxin Type

Address for correspondence: Sarah Adler, Centre for Documentation and Evaluation of Alternatives to Animal Experiments (ZEBET), Federal Institute for Risk Assessment (BfR), Berlin, Germany.

E-mail: Sarah.Adler@bfr.bund.de 
A for Injection (1), and the most promising approaches for their validation. Experts from industry, regulatory authorities, German ministries, academia, research, national and international validation centres, and animal welfare organisations, were invited to actively participate in the meeting.

The objective of the Expert Meeting was to review available alternative methods for BoNT potency testing, and to formulate recommendations for making progress toward implementing the Three Rs, i.e. Refinement, Reduction, and Replacement, in BoNT potency testing. In addition, ways in which communication on BoNT issues between manufacturers, researchers and regulators could be encouraged, and how improvements in regulatory harmonisation between different countries and continents could be achieved, were discussed. The meeting started with presentations by the individual participants, giving an overview on the regulatory and scientific status of alternative methods to the LD50 test for BoNT potency testing. Afterwards, the participants were divided into two separate break-out groups. Break-out Group 1 discussed the regulatory requirements for BoNT potency testing and the validation and implementation of alternative methods. Break-out Group 2 discussed the developed and available alternative methods and their suitability for reducing, refining or replacing the LD50 potency test.

\section{Introduction}

Botulinum neurotoxin is a biological product produced by the anaerobic, Gram-positive bacterium Clostridium botulinum. There are seven major and serologically distinct serotypes (A-G) of BoNT, characterised by a complex mode of action, which results in blockage of acetylcholine release at neuro-muscular nerve endings, leading to flaccid paralysis. This mechanism of toxicity comprises the four stages of binding, internalisation, translocation and proteolytic cleavage of a substrate, which leads to inhibition of synaptic exocytotoxic transmitter release. The different serotypes of BoNT act on different substrates.

BoNT-A and BoNT-B are available as licensed pharmaceuticals for the treatment of a variety of medical disorders, such as cervical dystonia, blepharospasm, spastic conditions and hyperhidrosis. However, BoNT is also used in so-called 'aesthetic medicine' to temporarily treat facial asymmetries or reduce facial lines. BoNT is of biological origin, and has an extremely high toxic pharmacological activity. Therefore, the safe and effective dosing of BoNT products requires accurate and reliable measurement of potency before batch release onto the market. The reference test for potency testing in the batch release of therapeutic BoNT, as well as for the detection of the toxin in clinical cases of botulism, is the mouse bioassay. In diagnostic testing, the serotype needs to be identified and the amount of toxin quantified. The detection of Clostridium botulinum in clinical samples, with subsequent identification and typing of the toxic culture supernatant by mouse bioassay, is often included in routine testing. This permits the identification of toxico-infections and delivers isolates for further testing and toxin-typing, either in further animal experiments, or by molecular methods.

In the manufacture of pharmaceutical BoNT products, the serotype and subtype of the toxin are well defined. The focus is on the biological activity of BoNT, which needs to be determined as accurately as possible, for the purposes of both safety and efficacy, to ensure that the correct potency of the product is supplied to clinicians for patient treatment. The current requirements of the EP for potency determination are based on standard practice, as approved in the marketing authorisations of products for the EU market, which stipulates that every production lot of BoNT must be tested in an LD50 potency test in mice. For a valid assay, the dilution range used should encompass the LD50 value. In practice, this can mean that at the highest dose, $90 \%$ of the animals are killed, and at the lowest dosage, $90 \%$ of the animals survive. Importantly, the precision of the test depends on dilution intervals and the number of animals used per concentration. The product dosage for patients is determined by the outcome of the potency assay.

In the case of BoNT LD50 potency testing, the dosing of animals is associated with severe suffering. Death is generally secondary to respiratory failure due to paralysis of the respiratory muscles. Consequently, the introduction of alternative methods is urgently required. A number of alternative tests have been developed in the past few decades, to replace the mouse bioassay. Additionally, promising methods are under development, which may, either alone or in combination with other assays, meet the rigid requirements of potency testing. To encourage movement away from the LD50 test, the possibility of using a validated in-house alternative to the LD50 assay has been included in the EP monograph (1).

\section{The Toxic Mechanism of BoNT}

BoNT induces muscle paralysis by blocking the release of acetylcholine at neuro-muscular nerve endings (2). BoNTs are composed of a $100 \mathrm{kDa}$ heavy chain and a $50 \mathrm{kDa}$ light chain $(3,4)$. The heavy chain is responsible for binding to a receptor located on the membrane of the neuron and translocation of the light chain, whereas the light chain acts as a highly specific proteolytic enzyme once internalised into the cell. BoNTs act via a 
complex mechanism (5) that comprises binding to cell surface, high affinity receptors of cholinergic nerve endings $(6,7)$, penetration into the plasma membrane by endocytosis (8), and $\mathrm{pH}$-dependent translocation of the light chain from the lumen of the vesicles into the cytoplasm. The light chain acts as a zinc-dependent protease that cleaves one or more components of a membrane fusion complex composed of syntaxin, SNAP-25 (a synaptosomalassociated protein) and synaptobrevin, thereby blocking exocytosis $(9,10)$. As a result of this cleavage event, the release of acetylcholine at the neuromuscular junction is inhibited, leading to a subsequent flaccid muscular paralysis.

\section{Previous Meetings on BoNT Potency Testing}

Two previous meetings were held in 2006, with the goal of promoting alternative methods to the LD50 potency test. These were: a meeting hosted by the European Directorate for the Quality of Medicines and Health Care (EDQM) of the Council of Europe; and a workshop in the USA organised by the US Interagency Coordinating Committee on the Validation of Alternative Methods (ICCVAM), the US National Toxicology Program (NTP) Interagency Center for the Evaluation of Alternative Toxicological Methods (NICEATM) and the European Centre for the Validation of Alternative Methods (ECVAM), in response to a nomination by the Humane Society of the United States (HSUS). The outcomes of these two meetings were reported and discussed at the ZEBET meeting in Berlin.

\section{The EDQM Meeting}

Catherine Milne, from the EDQM, reported on the Botulinum Toxin 3R Strategy Meeting, hosted in April 2006 by the EDQM, in Strasbourg, France. The participants included representatives of the three manufacturers of BoNT-A marketed in Europe, representatives from EU official national testing laboratories and licensing authorities, ECVAM and the EDQM. In addition, a conference call with authorities from the US Food and Drug Administration (FDA) Center for Drug Evaluation and Research (CDER), Health Canada, and ICCVAM was included, in order to permit the exchange of views in a more global context. The goal of the meeting was to look to the future for potency testing of BoNT in the context of the application of the Three Rs principles, supported by the relevant conventions of the Council of Europe and EU Directives.

The consensus was that the LD50 assay would remain the 'gold standard' for validation of alternative methods, but that this can only be used in a product-specific and method-specific setting due to method variability between laboratories, lack of common reference standards for the LD50 assay, and the need to maintain product-specific unitage. This should be taken into account as the alternative methods are being developed and validated.

It was agreed that in any replacement strategy, all the functions of the toxin (cell binding, internalisation, translocation and endopeptidase activity) would have to be assessed. This could be via a single assay or by a multi-assay approach.

A reduction strategy, involving a full assay approach for the bulk toxin (covering all three functions) and a simplified approach for the final product lot release, measuring a reduced set of criteria to monitor consistency, was considered to be worth investigating, as all lots would be derived from the same bulk toxin. Examples of this type of approach are already allowed in the EP, and are in use for human vaccines. The usefulness of this approach would be tightly linked to the definition of the robustness and reproducibility of the actual production process and the GMP controls in place.

The potential problems that might arise in the clinic if the unitage was changed, were a significant concern. The expression of units for alternative assays could be expressed in LD50 equivalents to avoid problems in the clinic. Possibilities for this would need to be further explored.

Based on the data presented, the product-specific aspects of the LD50 assay and the complications related to unitage, no clear candidate for a common method or reference was identified at that time. Nevertheless, the participants were open to the idea of exchanging reagents and data with the authorities, in order to facilitate development work, and the EDQM expressed a willingness to organise collaborative studies on the alternative methods in the context of the biological standardisation programme, once they had been further explored and established.

\section{The ICCVAM/NICEATM/ECVAM Workshop}

Martin Stephens, from the HSUS, reported on the ICCVAM/NICEATM/ECVAM Scientific Workshop on Alternative Methods to Refine, Reduce and Replace the Mouse LD50 Assay for Botulinum Toxin Testing, that was held in Silver Spring, MD, USA, in November 2006. About 115 participants, mostly from Europe and North America, participated in this workshop, including scientists from governmental and academic institutions, national and international regulatory authorities, industry, and the animal welfare community.

The consensus of the panel discussions at the ICCVAM/NICEATM/ECVAM Workshop (http:// iccvam.niehs.nih.gov/methods/biologics/bot_ workshop.htm) was that some of the alternative 
methods could be used in specific circumstances, or in a tiered-testing strategy, to reduce and refine the current LD50 assay. However, none of the methods reviewed could be used individually as a complete replacement for the mouse potency assay at that time. The panel discussions noted that, with additional development and validation efforts, one or more of the reviewed methods might be useful as a replacement for some of the current BoNT testing applications in the future. Finally, some best practices were discussed for decreasing the numbers of animals used, including the establishment of reference standards, the employment of standardised methodology, and reduction in the number of doses tested for assays when confirmation of potency is being evaluated.

It was reported at the 2009 Expert Meeting in Berlin that the US workshop in 2006 succeeded in bringing together international experts who work on disparate applications of the LD50 assay and alternatives for BoNT testing, and provided a snapshot of the status quo. It was less successful in fulfilling its stated goal of developing a set of strategic recommendations for future actions.

\section{The Regulatory Framework}

\section{Regulation on the protection of animals used for scientific purposes, according to Directive 86/609/EEC}

The protection of animals used for experimental and other scientific purposes is regulated in Europe by Directive $86 / 609 / E E C$, which is currently under revision (11). Among the aims of the directive is the reduction of the numbers of animals used for experiments, by requiring that an animal experiment should not be performed when an alternative method exists, and by encouraging the development and validation of alternative methods to replace animal methods. Furthermore, it requires the minimisation of any pain, suffering and distress caused to test animals. The Member States of the European Union have implemented the Directive $86 / 609 / E E C$ into their legislations. However, some of the member states, including Germany and the UK, have implemented national animal welfare acts that go beyond the requirements stated in Directive 86/609/EEC.

\section{Regulations for the marketing of pharmaceutical products}

\section{European regulations}

In Europe, the LD50 testing of BoNT, i.e. the bulk drug substance and the final drug product lot, is a requirement of the EP Monograph 2113, Botulinum Toxin Type A for Injection (1). EP monographs and texts are the legally binding quality standards for all medicinal products in the EU. The individual marketing authorisations for products marketed in the EU must apply these standards according to the EU Directive for Human Medicines 2001/83/EC (12), as amended by Directive 2004/27/EC (13).

The method currently used for the potency testing of all BoNT-A pharmaceutical products on the EU market, as accepted in the marketing authorisations by the European regulatory authorities, is the LD50 assay. Nevertheless, the specific monograph and the general notice open the possibility of developing alternative methods, in particular in the interest of animal welfare and the Three Rs. Since 2005, three different alternative approaches have been noted in the EP Monograph 2113, Botulinum Toxin Type A for Injection (1), but users are not limited to these methods, which are: "an endopeptidase assay in vitro, an ex vivo assay using the mouse phrenic nerve diaphragm, and a mouse bioassay using paralysis as the endpoint." Furthermore, it is stated in the EP that alternative methods must be suitably validated in comparison to the reference method, which is the LD50 assay: "After validation with respect to the LD50 assay (reference method), the product may also be assayed by other methods that are preferable in terms of animal welfare, including one of the above mentioned."

EP Monograph 2113, Botulinum Toxin Type A for Injection (1), is currently under consideration for revision. The revision provides additional details for a refined in vivo assay as an alternative to the LD50 potency assay that uses local paralysis as an endpoint. Moreover, a new monograph for BoNT type B for injection is under development, which presents a similar strategy for potency testing as for BoNT type A. The new and revised monographs were published in July 2009 in Pharmeuropa 21.3, for public consultation $(14,15)$.

\section{FDA regulations}

In the USA, FDA regulates BoNT products as drugs. The FDA requires the submission of data on BoNT potency, as well as on safety, purity, sterility and other parameters for these products. The regulations are specified in the Code of Federal Regulations 21 Chapter I (16). Although the FDA requires the appropriate potency testing of BoNT products, and specifies the performance attributes of such a test, it does not state which particular test should be used. While recognising that the international standard is the mouse LD50 potency assay, the FDA will also review data submitted in 
support of alternative potency assays and, when warranted, issue formal approval for their use.

\section{Quality Guidelines (ICH and EMA)}

The LD50 potency test is not only performed for release testing of BoNT, but also for other manufacturing purposes (Figure 1). For the marketing authorisation application, stability testing of drug substance and drug product is required. In addition, according to the principles of Good Manufacturing Practice (GMP), annual stability studies have to be performed after the medicinal products have been authorised. International (Europe, USA and Japan) Conference on Harmonisation of Technical Requirements for Registration of Pharmaceuticals for Human Use (ICH) Guideline Q5C, Stability Testing of Biotechnological/ Biological Products, requires, for products that are linked to a definable and measurable biological activity, that testing for potency should be part of the stability studies (17).

Furthermore, for the marketing authorisation application process in Europe, validation studies are required by the regulatory authorities of the Member States that demonstrate the suitability and reproducibility of the manufacturing process. The development of new formulations or products also requires additional process validation and stability studies, where LD50 tests are performed (ICH Q5E; 18).

\section{Validation Requirements for Alternative BoNT Potency Assays}

The basis for successful validation studies for alternative bioassays is $\mathrm{ICH}$ guideline Q2 Validation of Analytical Procedures (19). Since the LD50 tests of the different BoNT-producing companies vary and are product-specific $(20,21)$, the specific alternative methods have to be validated for each individual medicinal product. In this context, no standard validation programme can be applied, but individual validation plans have to be developed.

For the successful validation of an alternative assay, the specificity, reproducibility, precision, range and robustness have to be demonstrated. During the development and validation of the alternative method, sources of variability should be identified and variability should be minimised. The number of batches required for a successful validation and cross-validation with the LD50 test, and acceptable statistical limits, are key discussion points for validation requirements.

The Federal Institute for Drugs and Medical Devices (BfArM), and other European Regulatory Agencies, each offer the opportunity for companies to discuss their development plans and strategies at an early stage. These early discussions between manufacturers and regulatory authorities can be beneficial in facilitating the process of regulatory acceptance of alternative methods, and can help

\section{Figure 1: A schematic overview of the BoNT production process, demonstrating when potency tests are required to meet regulatory requirements}

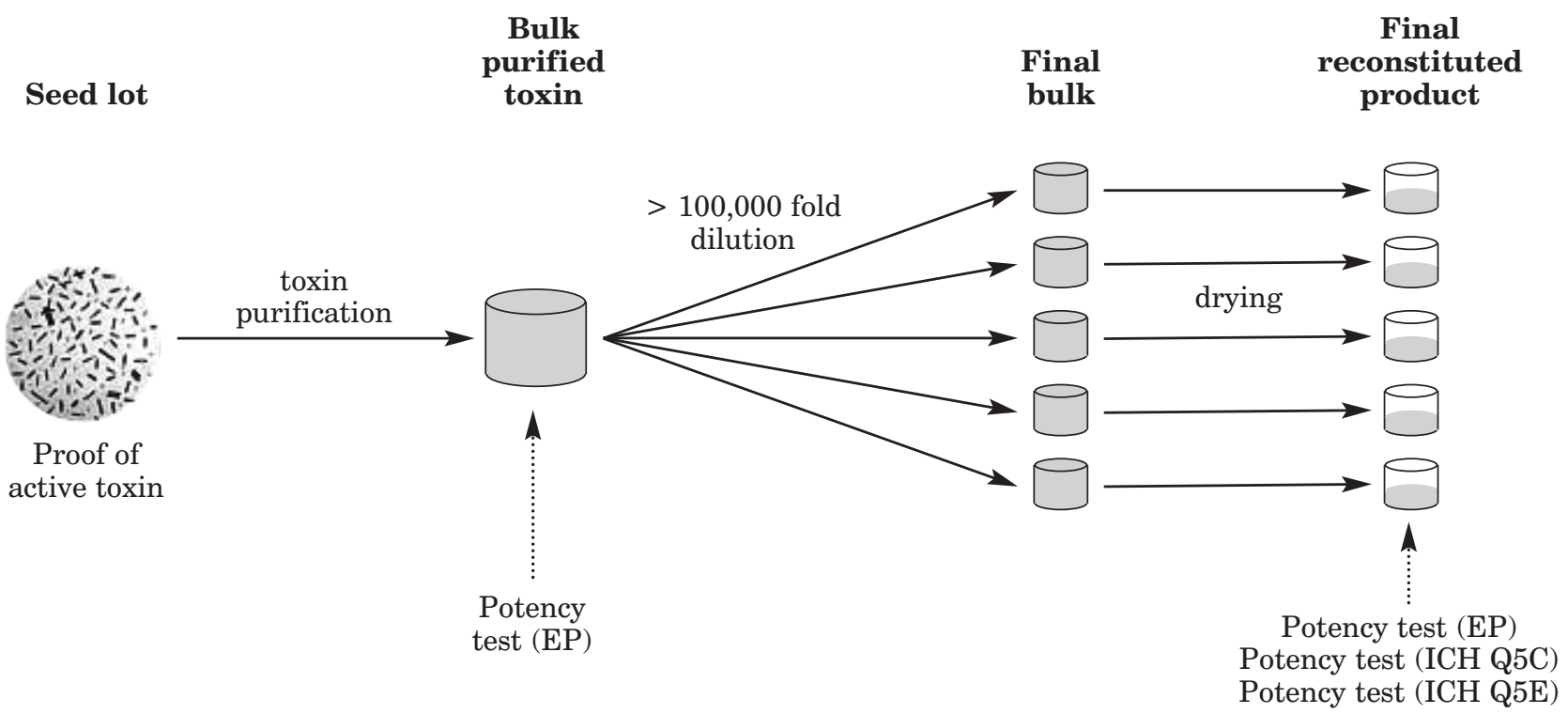

Potency tests are required in the EP for the bulk purified toxin and final reconstituted product. In addition, potency tests have to be performed according to the ICH, to demonstrate the product stability over time (ICH Q5C) and for process validation (ICH Q5E). 
manufacturers to plan appropriate validation studies on methods, such that they ultimately meet the regulatory requirements.

In this context, the question arose at the ZEBET Expert Meeting, of whether a general guidance document on the validation of alternative methods to the BoNT LD50 potency test would be helpful. However, the experts agreed that validation of such methods could not be generalised, since guidance for product-specific validation can only be given by the regulatory authorities in close communication with the manufacturers before and during the validation process, and will be specific for the particular product, the proposed alternative potency testing method used, and the data set generated.

Workshop participants from the regulatory authorities stated at the meeting that after the successful product-specific validation of a suitable alternative method and regulatory acceptance of a specific product, it would be easier for later applicants to achieve regulatory acceptance of the same method for use with other products. However, comprehensive data will need to be provided by each applicant. The experts also agreed that, from a pharmaceutical point of view, there would be no obvious reason why a method that could replace the LD50 potency test for one specific product should not work for the other products, as the BoNT-A medicinal products currently on the market do not differ in their modes of action.

\section{Alternative Methods in Product Stability and Process Validation}

There was discussion of whether alternative methods, unacceptable for product release alone (since they do not cover all functions of the toxin - e.g. the endopeptidase assay), could nonetheless be employed for other purposes, such as the monitoring of product stability or process validation. The participants had different opinions on this, but agreed that, in principle, it would be desirable to use the same assay for all testing requirements, i.e. stability, process validation and product release, in order to provide comparable data. Nevertheless, this issue was also discussed with regulatory experts. They stated that a reduced stability programme could be performed that could employ alternative assays, e.g. the endopeptidase assay, as a replacement for the LD50 potency assay for at least every second control test per year. Intermediate stability control tests would still have to be performed with the LD50 assay, or an alternative test reflecting fully functional activity of the toxin.

Such an approach might be acceptable in Europe, if sufficient data were available to provide information on the stability of the products.
However, it was mentioned that the lack of global harmonisation among regulatory authorities could be a problem.

\section{An International Reference Standard}

Due to the current lack of standardisation, the introduction of an international reference standard was discussed, to facilitate better comparison of different products and standardised unitage. The EP requires the use of in-house reference standards, as a common standard is currently unavailable. This is partly because of the differences observed with the LD50 assays used for the different products, which create difficulties in establishing such a standard. There have also been concerns over the problems that could arise in the clinic, if the existing unitage for dosing were changed for the individual products due to the use of a new reference unit. Nevertheless, it was argued that such a standard could help to promote the acceptance of alternative methods to the LD50 assay.

However, the majority of the participants suggested that the development of an international reference standard would be time-consuming and currently not necessary, since, for the acceptance of alternative potency assays, product-specific validations are required that would use the in-house standard. It was argued that the work on an international reference standard would currently delay validation efforts.

In contrast to the development of an international standard for the harmonisation of BoNT units, the experts recommended the development of BoNT reference materials that would be essential for comparing alternative potency assays and their performance in different laboratories. Reference materials already available at the National Institute for Biological Standards and Control (NIBSC) could be considered for interim use in planned method comparison studies (21).

\section{Harmonisation}

A main concern expressed at the meeting was the lack of global consensus between regulatory authorities on the implementation of alternative methods for BoNT potency testing. In Europe, the implementation of an alternative test method requires a variation application either at the national or at the European level. Moreover, the world-wide implementation of alternative methods for medicinal products is much more complex, and is considered to be an obstacle for globally-acting manufacturers. Some of the experts expressed their wish to promote international harmonisation and mutual acceptance criteria for the validation of alternative methods. 


\section{Establishment of a 'BoNT Expert Working Group'}

In order to overcome current difficulties in the development, optimisation, validation and regulatory acceptance of alternative methods to the BoNT potency test, the experts discussed the establishment of a 'BoNT Expert Working Group'. This group would deal with the essentials for acceptability, comparability and implementation of the Three Rs in BoNT potency testing. There was a common agreement to initially focus on the situation in Europe, as this could speed up the process of the implementation of alternative BoNT potency assays in this region.

A core group should be established, comprising experts from European regulatory authorities, Three $R_{s}$ and validation institutions, manufacturers and scientists. Experts from overseas should be invited as observers. In addition, a network of additional experts and stakeholders should be established, who would join the 'BoNT Expert Working Group' on a case-by-case basis. The Group should meet regularly up to three times per year during a proposed time-frame of four years, and should publish its recommendations on a regular basis.

The objectives of the 'BoNT Expert Working Group' would include, among others, providing advice and guidance on validation requirements for the proposed alternative methods, and defining minimum standards for a validation framework in order to implement the Three Rs in BoNT potency testing. The Group would assess replacement, refinement and reduction strategies, and review data, reference materials, methods and scientific approaches, and would promote the standardisation of protocols. The Group would also provide more transparency between the stakeholders and regulatory authorities.

All the attending participants expressed the wish that an independent institution should be responsible for the establishment and management of the 'BoNT Expert Working Group', and the BfR/ZEBET and BfArM agreed to accept this task.

\section{Alternative Methods to the LD50 Potency Test}

\section{Refinement assays}

There are a number of opportunities for refinement of the LD50 potency assay in the potency testing of BoNT. These could include, for example, the use of lower species, shortening of the duration of the procedure, and endpoints earlier than death, which would imply an increased frequency of animal inspections and intervention when death is impending, as has often been the practice with other lethal dose tests. In addition, refinement can be achieved by alternative experimental designs and analyses. To date, a number of these opportunities have been identified and exploited.

\section{Humane endpoints}

In the interests of the Three $\mathrm{Rs}_{\mathrm{s}}$, the conventional LD50 test was deleted from the OECD Test Guidelines Programme for the testing of chemicals in 2001 . It was replaced by three alternative methods, one of which includes the use of non-lethal humane endpoints (22). This deletion was only possible after weighing the risks and benefits, thus ensuring that all three alternative methods could serve the regulatory needs.

For BoNT, while complete deletion of the test is not yet possible, careful evaluation of the clinical signs that indicate imminent death has resulted in some success in implementing earlier (more humane) endpoints. Such signs include cyanosis, indrawn scaphoid abdomen, gasping, and behavioural responses such as immobility and social isolation. The application of such humane endpoints could be undertaken without affecting the results of the LD50 test, and data were presented that showed that up to $21 \%$ of the animals could be successfully culled by a humane method, rather than being allowed to die (Table 1). However, due to the fact that it is not yet feasible to establish such endpoints at a sufficiently early time-point (Figure 2), the current employment of humane endpoints does not permit a significant decrease in the suffering of the animals used.

In addition, the above-mentioned clinical signs that should indicate imminent death have not yet been sufficiently investigated. A change from the lethal endpoint to the refined humane endpoints would also require a product-specific validation

\section{Table 1: The results of experimental studies that applied humane endpoints}

\begin{tabular}{lc}
\hline Study number & \% Humane endpoint \\
\hline 1 & $16 \%$ \\
2 & $11 \%$ \\
3 & $11 \%$ \\
4 & $15 \%$ \\
5 & $15 \%$ \\
6 & $21 \%$ \\
\hline Average & $15 \%$ \\
\hline
\end{tabular}

$\%$ Humane endpoint specifies the percentage of mice that could have been culled by a humane method rather than going on to die, without affecting the results of the LD50 test. 
Figure 2: The numbers of dead mice over time, following BoNT injection

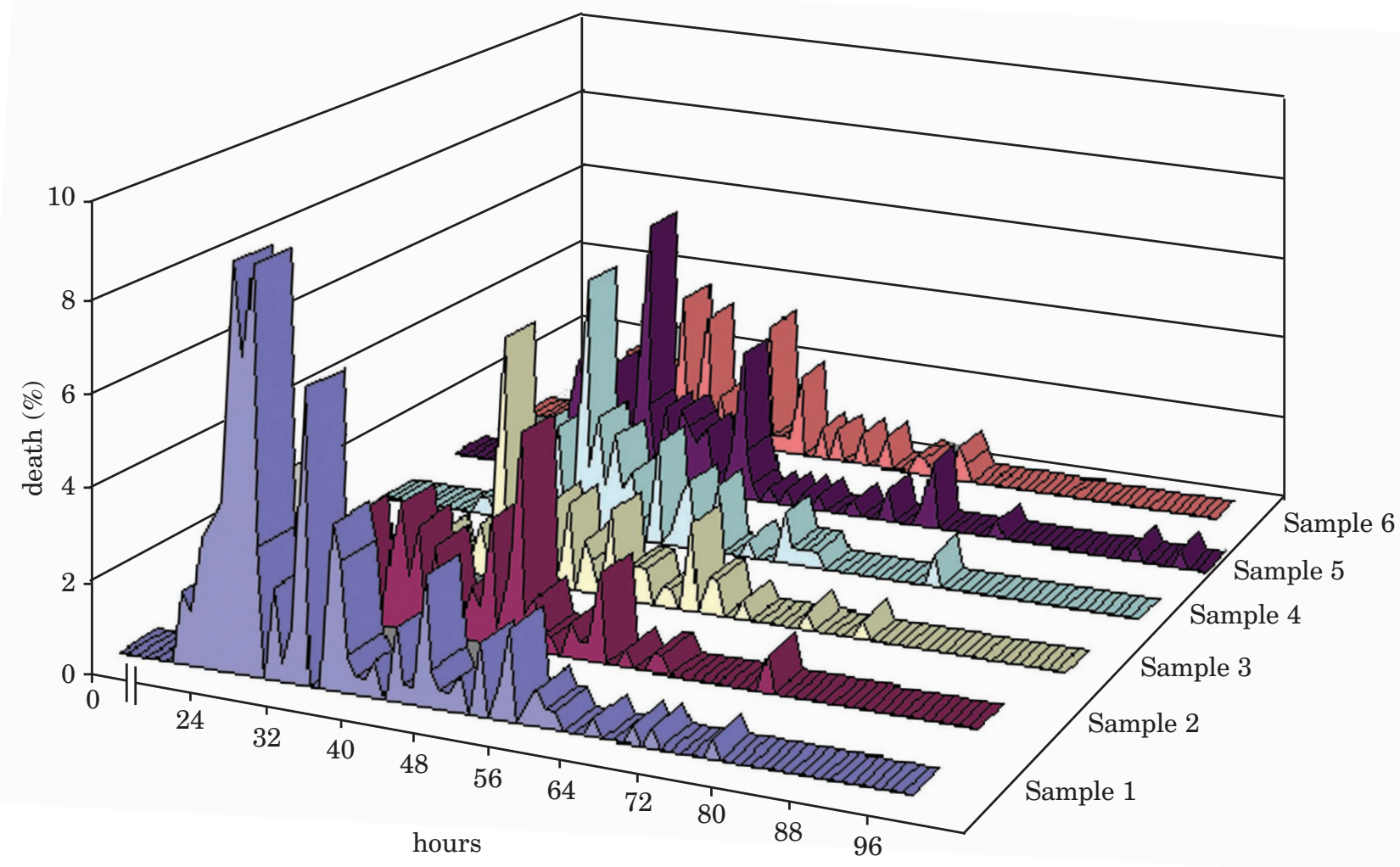

The graph shows the mean results of six independent runs.

study, and most experts recommend that further effort should not be invested in the development of refined assays based on humane endpoints at present, but that the focus should be on other refinement or replacement assays.

\section{Local paralysis assays}

Changing the LD50 potency assay to an alternative, less severe, test by using non-lethal endpoints, is complicated. Such alternative tests include the mouse flaccid paralysis assay (23), the rat muscle force (RMF) assay (24), the digital abduction score (DAS) assay $(25,26)$, and the rat gastrocnemius assay. From a Three Rs point of view, these assays would be preferred to the LD50 potency test, as only sub-lethal doses of the toxin are injected and animals generally fully recover from the treatment, even at the highest dose used.

In the flaccid paralysis assay, different sublethal concentrations of BoNT are injected subcutaneously into the top of the left hind leg, the inguinocrural region of the lower abdomen of a mouse. The animals are then scored by two or more observers according to the size of the local abdom- inal bulge (Figure 3) at 24 and 48 hours, by using a five-point scale (Table 2). This scoring system was introduced because the method had been previously criticised for its subjective and non-quantitative read-out.

The dose-response, suitable for calculation of potency, is obtained by plotting the scores against the log toxin dose for the test and reference samples. Then the relative potency is calculated by a parallel line method. This approach has been used at the NIBSC, primarily to confirm activity in product-specific reference preparations. At the NIBSC, the LD50 test has not been performed for more than 10 years (27).

The advantages of the flaccid paralysis assay are not only a refined endpoint, but also ease of use, fast one-off read-out (48 hours), and increased sensitivity with comparable precision, coupled with the use of only $20 \%$ of the number of animals used in a comparable LD50 test. Furthermore, the dosing is considered more relevant to clinical use, as muscle paralysis rather than systemic toxicity is evaluated. Recent potency data presented by the NIBSC confirmed an excellent agreement with LD50-determined potency for seven different therapeutic products. 


\section{Figure 3: The mouse flaccid paralysis assay}
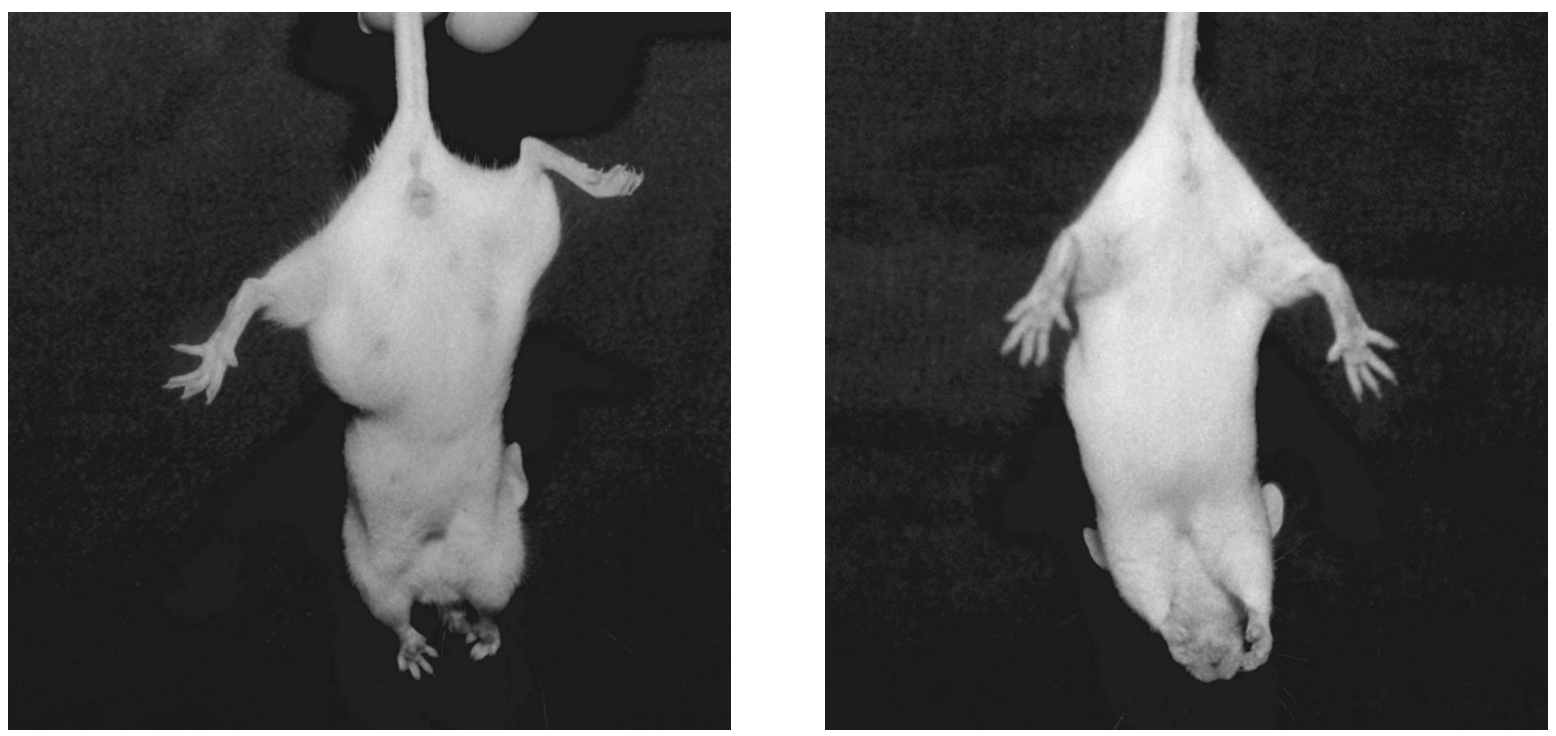

The mouse on the left has been injected with BoNT to assay the potency of a test sample, and shows an abdominal bulge at the site of injection (stage 3, Table 2), compared with the uninjected control mouse on the right (stage 0 , Table 2) (illustrations provided by D. Sesardic et al. [29]).

However, criticism was expressed by some experts during the meeting. One of the concerns was that, although local paralysis was a relevant endpoint, the scoring could still be too subjective. Furthermore, it was mentioned that the low precision of $20 \%$ which was the rate achieved with an $80 \%$ reduction in animal number - may necessitate an increase in the number of test animals used, so that the number of animals necessary may eventually be close to that for the LD50 test. The performance of a validation study involving several laboratories, to confirm the precision of the test that is referred to in the EP, was considered to be a useful proposal.

The experts also discussed the rat muscle force (RMF) assay, the digital abduction score (DAS) assay and the rat gastrocnemius assay. All three methods were evaluated as being not yet suitable for BoNT potency testing, and the RMF assay and the rat gastrocnemius assay were recommended only for research purposes.

\section{Replacement assays}

Ex vivo assays

1. The mouse hemidiaphragm assay (HDA): The HDA (28) operates with an isolated nerve muscle preparation (Figure 4). When BoNT is added to the organ bath in which the muscle has been

\section{Table 2: An example of a recommended scoring scheme that is used to define the response of individual mice in the flaccid paralysis test}

\begin{tabular}{ll}
\hline Stage & Description \\
\hline $\mathbf{0}$ & No signs, normal \\
\hline $\mathbf{1}$ & Just detectable bulge, e.g. covering an area of approximately $0.5 \mathrm{~cm}$ in diameter, or less. \\
\hline $\mathbf{2}$ & $\begin{array}{l}\text { More pronounced bulge, e.g. covering an area greater than } 0.5 \mathrm{~cm} \text { in diameter, but less than the } \\
\text { maximum radius of the hind leg heel. }\end{array}$ \\
\hline $\mathbf{3}$ & $\begin{array}{l}\text { More extensive bulge extending over a larger area. Extending below hips and top of thigh when viewed } \\
\text { from the side and beyond the maximum radius. }\end{array}$ \\
\hline $\mathbf{4}$ & $\begin{array}{l}\text { Maximal local effect. More extensive bulge extending over a larger area. Will often extend as far as the } \\
\text { bottom of the rib cage, or over a large area with extensive distension or bulging. }\end{array}$ \\
\hline
\end{tabular}


placed, the contraction amplitude of the nervestimulated muscle gradually declines until it disappears. The contraction of the diaphragm is recorded isometrically, by using a force transducer, and the contraction amplitude is assessed over time. The time at which the muscle is paral- ysed by $50 \%$ of the original contraction amplitude is determined, following the application of different concentrations of BoNT. The recorded time of the onset of $50 \%$ paralysis $\left(t_{1 / 2}\right)$ decreases with increasing concentrations of BoNT.

The advantages of the HDA are fast read-out (a

Figure 4: The hemidiaphragm assay

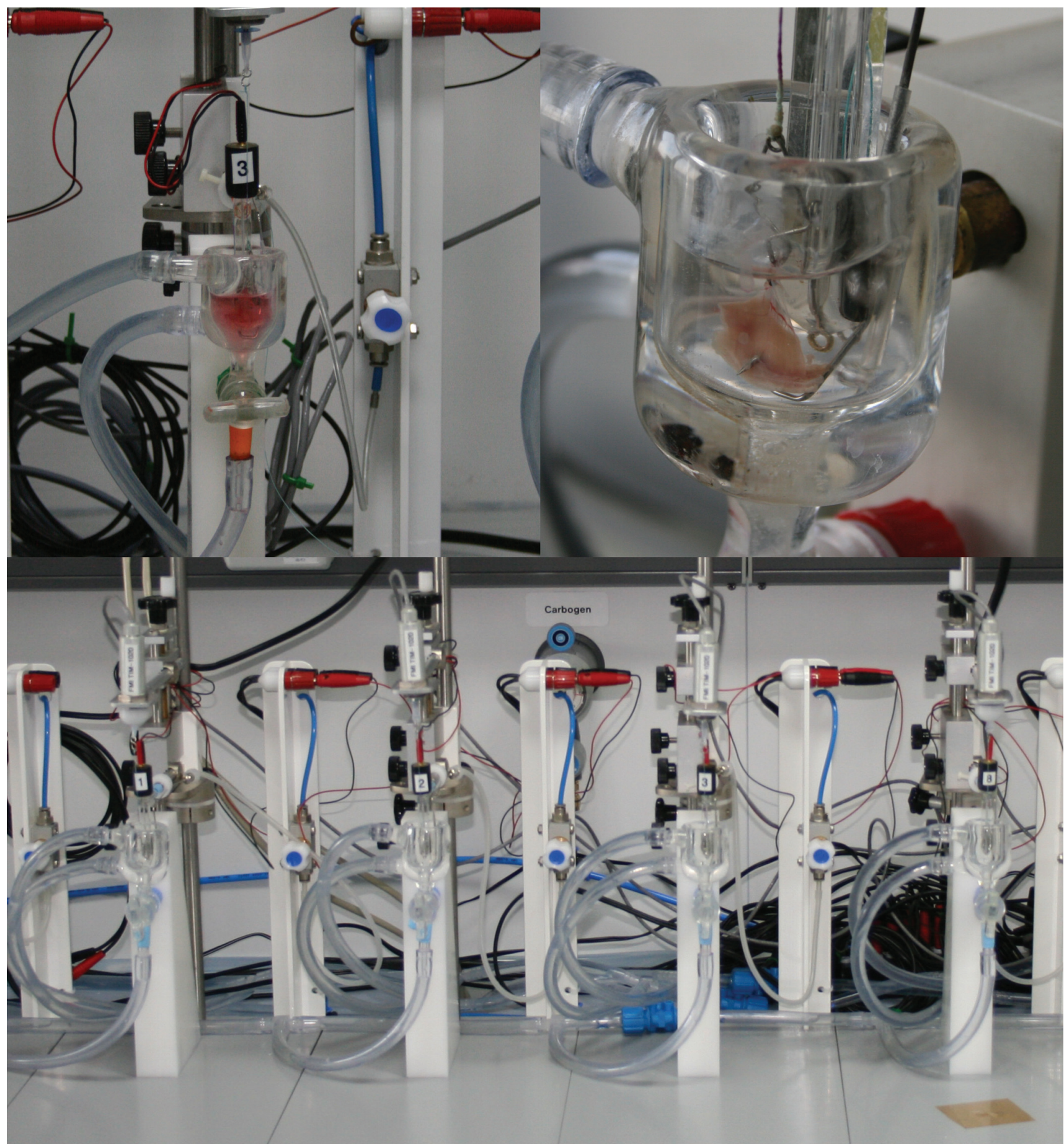

The hemidiaphragm assay operates with an isolated nerve muscle preparation that is placed in an organ bath. The contraction of the diaphragm is recorded isometrically, by using a force transducer, and the contraction amplitude is assessed over time (illustrations provided by H. Bigalke). 
few hours) and full functionality. This is an organotypic assay — which means that the animals are humanely killed, and the determination of BoNT potency is then carried out on the isolated nerve muscle preparation. The method is technically challenging and requires sophisticated and expensive equipment. The robustness of the assay was tested at the Medical School of Hannover, in the presence of human serum with various protein and lipid concentrations. The test was validated for measurement of the presence of antibodies to neurotoxin in sera from BoNT-treated patients, and was shown to fulfil the criteria of specificity, selectivity and precision for this purpose. A validation study for BoNT potency testing by using the HDA began in 2009, in collaboration with Merz Pharmaceuticals.

The discussions at the Expert Meeting revealed a number of questions that could not be answered at present, e.g. the product specificity of the assay, the strain specificity of the ex vivo material, and the comparability of the steepness of the concentration-response curves of the HDA and the LD50 assay. Recent published data from the NIBSC identified improved precision with an inbred mouse strain, but also observed supplierspecific differences in A toxin activity in the HDA (29).

\section{The intercostal neuromuscular junction (NMJ)} assay: Another ex vivo assay discussed at the Expert Meeting was the intercostal NMJ assay, which uses ex vivo rat intercostal muscle preparations (30). As described above for the HDA, this ex vivo assay still requires the killing of animals (rats), but successfully addresses the question of their suffering. Up to six individual rat intercostal rib sections can be obtained from a single rib cage. Upon treatment with BoNT injected directly into the tissue sections, the contractile response is monitored upon electrostimulation of the intercostal nerve over time. The NMJ assay was evaluated by the experts as an assay that employs a relevant endpoint and reduces the suffering of the animals used. However, a pre-validation study with this assay, performed by one manufacturer, was unsuccessful. The experts at the meeting considered that this might have been due to the relatively small size of the muscle preparations. The tissue size employed in the HDA, for example, is substantially bigger, and the phrenic nerve is a relatively robust nerve.

\section{In vitro assays}

1. Cellular assays: Potency assays based on cells offer an advantage over other in vitro models, as they provide information on all the essential steps in the BoNT intoxication process, i.e. binding, internalisation and intracellular activity. In theory, only the cell-based assays have the potential to fully replace animal-based potency tests for all applications. Furthermore, they could be applied to all stages of the production process, including the testing of the purified bulk active toxin component.

Despite active research in this field, no suitable assays with the desired sensitivity and characteristics for the potency assessment of toxin-based products are yet available. Primary neuronal cells, derived from rat spinal cords, have been extensively studied and confirmed to offer the desired sensitivity. However, robustness and reproducibility have been limiting factors so far in the introduction of such methods within a quality control setting. Human cell lines of neuronal lineages would provide the best option in the testing of BoNT potency, but, up to now, none of the available cell lines have offered the required sensitivity, unless they were fully differentiated to neuronal cell types.

A number of cell-based assays for potency testing are currently under development by experts from both the NIBSC and Allergan, Inc. Strategies at the NIBSC have focused on the differentiated human neuroblastoma cell line, SH-SY5Y. With these cells, the inhibition of depolarisation-evoked neurotransmitter release is measured after incubation with botulinum toxin. In addition, collaborative studies with the NIBSC Scientific Division of Cell Biology and Imaging (CBI), have provided the opportunity to study differentiated cell lines and human stem cells that were grown in multielectrode dishes. With such dishes, the spontaneous electrical activity of the cells can be recorded before and after BoNT exposure. Although these approaches showed increased sensitivity to picomolar levels of BoNT, it was anticipated at the meeting that they are not sufficiently robust for quality control testing, but are likely to remain useful research tools.

Another strategy focused on by the NIBSC, was the development of a new generation cell-based endopeptidase assay that employs antibody reagents already used in the SNAP-25 ELISA format. In addition, new antibodies were generated for the capture and detection of SNAP-25, and an optimum protocol for the sensitive capture of recombinant SNAP-25 and cleaved SNAP-25 from cell lysates was developed. The sensitivity of the method was confirmed in the fully-differentiated human neuronal cell line, SH-SY5Y, although primary rat spinal cord cells offered greater sensitivity in Western blotting (31) and immunodetection of cleared SNAP-25 (unpublished data). However, robustness and precision in comparison to the conventional LD50 assay have not yet been assessed, so further studies are necessary.

Allergan Inc. have developed a novel cell-based screening Western blot assay for measuring BoNT 


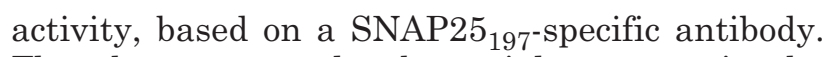
They have reported substantial progress in the optimisation of the assay, resulting in increased sensitivity and improvements in assay read-out.

Another ongoing project, involving collaboration between the University of Veterinary Medicine Hannover and the Georg-August University of Göttingen, focuses on the development of a BoNT testing assay based on the human teratocarcinoma cell line, Ntera2 (NT-2). These cells can be differentiated into fully functional, post-mitotic neurons, which display a variety of neurotransmitter phenotypes (Figure 5; 32). In this project, the process to generate post-mitotic neurons was significantly shortened to approximately four weeks' duration (33). During differentiation, the NT-2 acquired typical neuronal markers, such as beta-tubulin type III, MAP-2 and phosphorylated tau, and grew neural processes which expressed punctate immunoreactivity for synapsin and synaptotagmin, suggesting the formation of synaptic structures. A major subset of the model neurons showed immunoreactivity to the cholinergic markers, choline acetyltransferase and vesicular acetylcholine transporter. Moreover, spontaneous postsynaptic currents in glia-free cell cultures were detected, which result from the firing of excitatory and inhibitory NT-2 neurons (34). These spontaneously-active neural networks may be a useful tool in the detection of BoNT potency in blocking neurotransmission, by using cell physiological techniques.

The experts have extensively reviewed the use of cell-based assays, in order to determine acceptable cell types for the BoNT potency assays. They agreed that primary neuronal cells are not favoured from a regulatory point of view, as they are too variable. No primary cells are currently being used by industry to develop new BoNT assays. Cell lines, on the other hand, are easier to standardise than primary neuronal cells, but often are not as sensitive when used in assays. If used in BoNT potency assays, cell lines must first be very well characterised.

In addition, the experts discussed the cell types that would be best suited for testing the potency of BoNT. They expressed different opinions about the suitability of neuronal cells, either alone or in combination with muscle cells. Some experts thought that cells applied in a potency assay should resemble, as closely as possible, motor neurons. However, other experts believed that any cells that could reflect the action of BoNT, from receptor binding, to SNAP-25 cleavage or blocking of trans-

\section{Figure 5: The in vitro differentiation of NT-2 cells into post-mitotic neurons}

a)

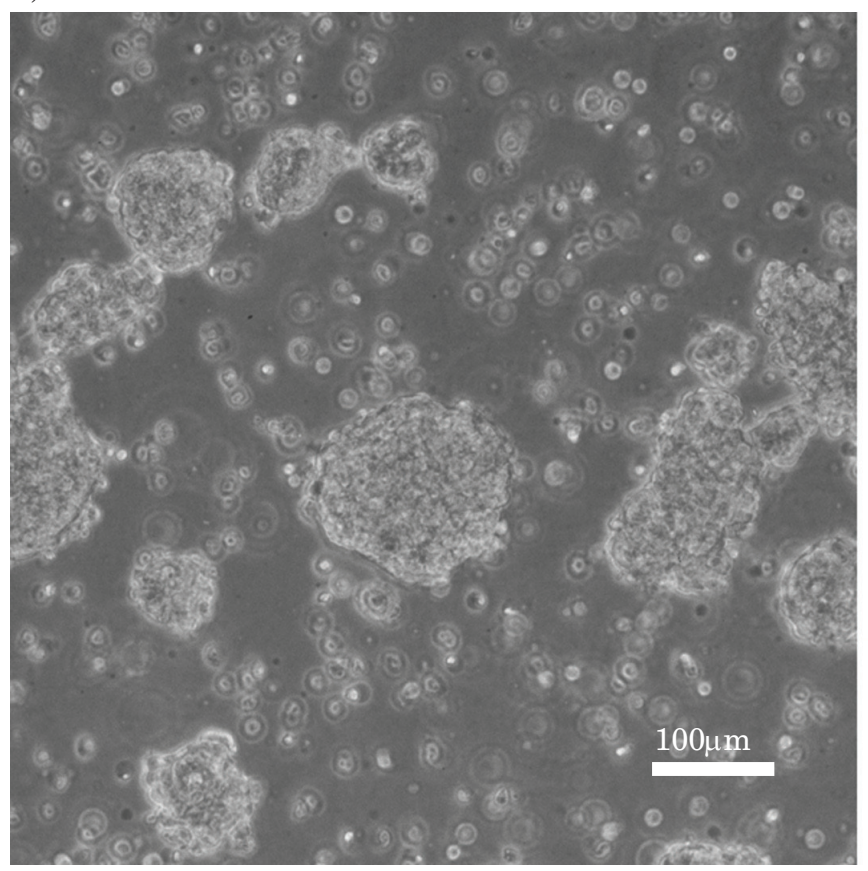

b)

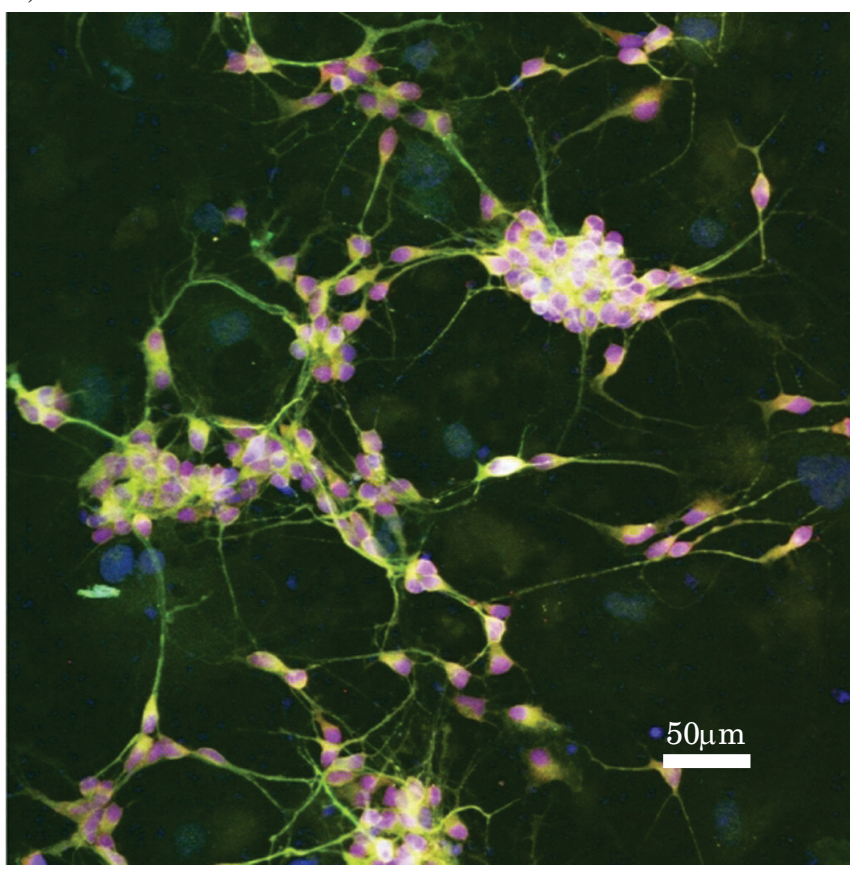

a) Expansion of NT-2 cells in free floating cell spheres. This technique resembles the sphere culture method used in embryonic stem cell differentiation. b) Post-mitotic NT-2 neurons, showing the immunofluorescent staining of a neuronal marker. Spindle shaped cell bodies send out processes that form a neural network (illustrations provided by G. Bicker, H. Böhnel and F. Gessler). 
mitter release, would be useful. In addition, the importance of the species of origin of the cells was discussed. The question arose as to whether neuromuscular junctions should be cholinergic in a BoNT potency test, or could be also glutaminergic, as, for example, found in some lower species. Some experts thought it would be fundamental for a test to be based on a system with cholinergic neuromuscular junctions. However, others thought that the transmitters would not be of importance, but solely the substrate, SNAP-25, as BoNT would also act on different types of glands.

There are a number of challenges associated with the use of cellular BoNT potency assays. The experts agreed that a key problem with the cellbased assays currently under investigation, was the relatively flat dose-response curves seen, in comparison with the LD50 assay. This observation was explained by the minimum signal resulting from a cell due to the low amount of transmitters released. This effect is amplified in vivo or in organotypic assays, due to the muscle activity, especially in the voltage-dependent channels.

A steep concentration-response curve was considered necessary, as a good separation of the tested concentrations is of great importance. The EP monograph requires a potency specification between $80 \%$ and $125 \%$ (1). Therefore, three to five concentrations have to be tested in this range.

The experts discussed how the cell signals could be amplified in vitro. They agreed that transgenic cell lines, e.g. Green Fluorescent Protein (GFP)expressing cell lines or cells over-expressing receptors or substrate, could result in steeper concentration-response curves and therefore provide suitable in vitro models for BoNT potency testing. In addition, the incubation time of BoNT in the potential assay will have an influence on the steepness of the concentration-response curve.

Finally, it was emphasised by the experts that cells are the minimum accepted unit that can cover all the steps of the BoNT mechanism of toxicity. To go beyond this level to molecular assays would require a combination of different test methods. However, no cell-based assays are as yet at a stage appropriate for a validation study.

\section{Molecular assays}

\section{Endopeptidase assays}

The information on the intracellular mode of action of BoNTs provided the opportunity to develop enzyme cleavage assays. A series of papers were published, in which endopeptidase assays were applied to BoNT drug products (35-37). The approach has been validated in-house at the NIBSC for a particular product, and has been in use for the last five years, to confirm the manufacturer's claim of potency against a product-specific reference preparation. The NIBSC reported that, by using the endopeptidase assay, it was possible to detect nanogramme quantities of toxin in the presence of high concentrations of bulking agents and stabilisers in the final product, with superior sensitivity and excellent agreement with the mouse LD50. Furthermore, the assay afforded a high precision that was superior to that of the in vivo models and, in addition, ease of use, robustness and ready transferability. The NIBSC has recently improved the assay protocol (38), and now considers this to be an excellent method for the evaluation of trends and consistency.

The method has been criticised for its inability to reflect all the functional domains of the toxin known to contribute to the in vivo activity, as only the light chain activity is measured. The experts agreed that it could be promising in support of potency testing for process validation and stability, but might not be suited for product release. On the other hand, some experts considered the endopeptidase assay to be suitable for assigning the potency of the final product after successful product-specific validation, if the potency of the final bulk was assessed by an assay able to cover the whole toxic mechanism of BoNT.

\section{Binding and translocation assays}

Binding and translocation assays are available, but are still at the research stage. Ipsen, for example, has developed a ganglioside binding assay. The reagents for such assays are not yet commercially available and may be of limited use, in view of the fact that gangliosides might not be high affinity receptors for the toxins.

\section{Combination of molecular assays}

Due to the fact that molecular assays can only provide information on single steps in the toxicological mechanism of BoNT, various tests would have to be combined in order to provide all the necessary information on potency. The experts discussed how different assays could be combined, and agreed that this would be a very difficult task, as different tests would provide different potency values (units) that would have to be linked or weighted in some way.

\section{Public Funding}

The experts recommended increased funding on research into cellular neurotoxicity mechanisms, in order to identify relevant mechanisms for novel 
assays, and into feasibility and prevalidation studies of existing methods. Furthermore, it was reported that funding possibilities exist from the German Federal Ministry of Education and Research, and that also the US National Institute of Health is currently funding small enterprises for the development of alternative assays for BoNT potency testing.

\section{Reduction of Animal Numbers and Refinement of the LD50 Assay}

Allergan and Ipsen reported a substantial reduction in the numbers of animals used in the LD50 potency test. Allergan have improved their dosing range, which has resulted in the testing of fewer doses and the requirement for fewer animals per dose. By using this approach, they could reduce the animal numbers by 50\%. Ipsen stated that they have reduced the testing time from 96 to 72 hours, which resulted in a reduction of suffering (refinement). Merz informed the participants of the meeting that these approaches have also been in practice at their facilities, since the beginning of their own BoNT product development.

\section{Conclusions and Recommendations}

1. The industry and scientists have made progress in implementing reduction and refinement in BoNT potency testing, and in the development of alternative potency assays.

2. General guidance on the product-specific validation of alternative methods for BoNT potency methods cannot be provided. Guidance on product-specific validation is given continuously by regulatory authorities to manufacturers, both before and during the validation process.

3. Regulatory authorities should actively be involved in international harmonisation and in defining mutual acceptance criteria for alternative methods to LD50 potency testing.

4. A 'BoNT Expert Working Group' should be established, to provide advice on validation requirements for alternative methods, define minimum standards for a validation framework, assess refinement and reduction strategies, review data, test methods, reference materials and scientific approaches, and promote the standardisation of protocols. BfR/ZEBET, in collaboration with BfArM, will be responsible for establishing the 'BoNT Expert Working Group'.

5. Funding should be made available and its use coordinated, to develop and validate alternative methods for BoNT potency testing according to the Three Rs principles. In particular, the development of a replacement alternative for BoNT testing should have the highest priority.

6. Reference materials should be developed to promote the comparability of different BoNT potency assays.

7. Alternative methods that are not yet able to replace the LD50 test in all steps of the production process may still be useful in testing for product stability, process validation or lot release.

\section{References}

1. Council of Europe (2006). Botulinum Toxin Type A for Injection 01/2005:2113. In European Pharmacopoeia 6.0, pp. 1117-1119. Strasbourg, France: Council of Europe.

2. Simpson, L.L. (1981). The origin, structure, and pharmacological activity of botulinum toxin. Pharmacological Reviews 33, 155-188.

3. Lacy, D.B. \& Stevens, R.C. (1997). Recombinant expression and purification of the botulinum neurotoxin type A translocation domain. Protein Expression \& Purification 11, 195-200.

4. Hanson, M.A. \& Stevens, R.C. (2000). Cocrystal structure of synaptobrevin-II bound to botulinum neurotoxin type $\mathrm{B}$ at $2.0 \AA$ resolution. Nature Structural Biology 7, 687-692.

5. Simpson, L.L. (1980). Kinetic studies on the interaction between botulinum toxin type $\mathrm{A}$ and the cholinergic neuromuscular junction. Journal of Pharmacology \& Experimental Therapeutics 212, 16-21.

6. Dolly, J.O., Black, J., Williams, R.S. \& Melling, J. (1984). Acceptors for botulinum neurotoxin reside on motor nerve terminals and mediate its internalization. Nature, London 307, 457-460.

7. Simpson, L.L. (1986). Molecular pharmacology of botulinum toxin and tetanus toxin. Annual Review of Pharmacology \& Toxicology 26, 427-453.

8. Black, J.D. \& Dolly, J.O. (1986). Interaction of 125Ilabeled botulinum neurotoxins with nerve terminals. II: Autoradiographic evidence for its uptake into motor nerves by acceptor-mediated endocytosis. Journal of Cell Biology 103, 535-544.

9. Schiavo, G., Rossetto, O., Santucci, A., DasGupta, B.R. \& Montecucco, C. (1992). Botulinum neurotoxins are zinc proteins. Journal of Biological Chemistry 267, 23,479-23,483.

10. Schiavo, G., Matteoli, M. \& Montecucco, C. (2000). Neurotoxins affecting neuroexocytosis. Physiological Reviews 80, 717-766.

11. Anon. (1986). Council Directive 86/609/EEC of 24 November 1986 on the approximation of laws, regulations and administrative provisions of the Member States regarding the protection of animals used for experimental and other scientific purposes. Official Journal of the European Communities L358, 18/12/1986, 1-28. Available at: http://eur-lex. europa.eu/smartapi/cgi/sga_doc?smartapi!celexplus!prod!DocNumber\&lg=en\&type_doc=Directive \&an_doc=86\&nu_doc=609 (Accessed 22.06.10). 
12. Anon. (2001). Directive $2001 / 83 / E C$ of the European Parliament and of the Council of 6 November 2001 on the Community code relating to medicinal products for human use. Official Journal of the European Communities L311, 28/11/2001, 67-128. Available at: http://eur-lex.europa.eu/LexUriServ/ LexUriServ.do?uri=CELEX:32001L0083:EN:NOT (Accessed 24.06.10).

13. Anon. (1996). Directive 2004/27/EC of the European Parliament and of the Council of 31 March 2004 amending Directive 2001/83/EC on the Community code relating to medicinal products for human use. Official Journal of the European Communities L136, 30/04/2004, 34-57. Available at: http://eur-lex.europa.eu/LexUriServ/LexUriServ. do?uri=OJ:L:2004:136:0034:0057:EN:PDF (Accessed 05.05.10)

14. Anon. (2009). Botulinum toxin type A for injection. Pharmeuropa 21.3, 361-363.

15. Anon. (2009). Botulinum toxin type B for injection. Pharmeuropa 21.3, 363-365.

16. FDA (undated). The Code of Federal Regulations. Title 21 - Food and Drugs. Chapter I - Food and Drug Administration, Department of Health and Human Services. (4-1-10 Edition). Available at: http://www.access.gpo.gov/nara/cfr/waisidx_10/21cf rv1_10.html (Accessed 10.08.10).

17. ICH (1996). ICH Harmonised Tripartite Guideline. Quality of Biotechnological Products: Stability Testing of Biotechnological/Biological Products, Q5C, 10pp. Geneva, Switzerland: International Conference on Harmonisation of Technical Requirements for Registration of Pharmaceuticals for Human Use. Available at: http://www.ich.org/LOB/ media/MEDIA427.pdf (Accessed 24.06.10).

18. ICH (2005). ICH Harmonised Tripartite Guideline. Comparability of Biotechnological/Biological Products Subject to Changes in Their Manufacturing Process, Q5E, 16pp. Geneva, Switzerland: International Conference on Harmonisation of Technical Requirements for Registration of Pharmaceuticals for Human Use. Available at: http://www.ich.org/LOB/ media/MEDIA1196.pdf (Accessed 24.06.10).

19. ICH (1994). ICH Harmonised Tripartite Guideline. Validation of Analytical Procedures: Text and Methodology, Q2(R1), 17pp. Geneva, Switzerland: International Conference on Harmonisation of Technical Requirements for Registration of Pharmaceuticals for Human Use. Available at: http://www.ich.org/LOB/media/MEDIA417.pdf (Accessed 24.06.10).

20. McLellan, K., Das, R.E., Ekong, T.A. \& Sesardic, D. (1996). Therapeutic botulinum type A toxin: Factors affecting potency. Toxicon: Official Journal of the International Society on Toxinology 34, 975-985.

21. Sesardic, D., Leung, T. \& Gaines Das, R.E. (2003). Role for standards in assays of botulinum toxins: International collaborative study of three preparations of botulinum type A toxin. Biologicals 31, $265-276$

22. OECD (2001). OECD Guideline for the Testing of Chemicals. No. 420. Acute Oral Toxicity: Fixed Dose Procedure, 14pp. Paris, France: Organisation for Economic Co-operation and Development. Available at: http://lysander.sourceoecd.org/vl=942322/cl=12/ $\mathrm{nw}=1 / \mathrm{rpsv} / \mathrm{cw} /$ vhosts/oecdjournals/1607310x/v1n4/ contp1-1.htm (Accessed 24.06.10).

23. Sesardic, D., McLellan, K., Ekong, T.A.N. \& Gaines Das, R. (1996). Refinement and validation of an alternative bioassay for potency testing of therapeutic botulinum type A toxin. Pharmacology \& Toxicology 78, 283-288.

24. Pickett, A., O'Keeffe, R., Judge, A. \& Dodd, S. (2008). The in vivo rat muscle force model is a reliable and clinically relevant test of consistency among botulinum toxin preparations. Toxicon: Official Journal of the International Society on Toxinology 52, 455-464.

25. Aoki, K.R. (2001). A comparison of the safety margins of botulinum neurotoxin serotypes $\mathrm{A}, \mathrm{B}$, and $\mathrm{F}$ in mice. Toxicon: Official Journal of the International Society on Toxinology 39, 1815-1820.

26. Aoki, K.R. (2002). Botulinum neurotoxin serotypes $A$ and $B$ preparations have different safety margins in preclinical models of muscle weakening efficacy and systemic safety. Toxicon: Official Journal of the International Society on Toxinology 40, 923-928.

27. Sesardic, D. \& Gaines Das, R.E. (2009). Botulinum toxin: Applying the 3Rs to product potency testing, 8pp. London, UK: NC3Rs. Available at: http://www. nc3rs.org.uk/downloaddoc.asp?id=853\&page $=1023$ \&skin=0 (Accessed 24.06.10).

28. Wohlfarth, K., Göschel, H., Frevert, J., Dengler, R. \& Bigalke, H. (1997). Botulinum A toxins: Units versus units. Naunyn-Schmiedeberg's Archives of Pharmacology 355, 335-340.

29. Rasetti-Escargueil, C., Jones, R.G., Liu, Y. \& Sesardic, D. (2009). Measurement of botulinum types A, B and E neurotoxicity using the phrenic nerve-hemidiaphragm: Improved precision with inbred mice. Toxicon: Official Journal of the International Society on Toxinology 53, 503-511.

30. Huber, A., France, R.M., Riccalton-Banks, L., McLaren, J., Cox, H., Quirk, R.A., Shakesheff, K.M., Thompson, D., Panjwani, N., Shipley, S. \& Pickett, A. (2008). The Intercostal NMJ Assay - A new alternative to the conventional LD50 assay for the determination of the therapeutic potency of botulinum toxin preparations. ATLA 36, 141-152.

31. Pellett, S., Tepp, W.H., Clancy, C.M., Borodic, G.E. \& Johnson, E.A. (2007). A neuronal cell-based botulinum neurotoxin assay for highly sensitive and specific detection of neutralizing serum antibodies. FEBS Letters 581, 4803-4808.

32. Podrygajlo, G., Tegenge, M.A., Gierse, A., PaquetDurand, F., Tan, S., Bicker, G. \& Stern, M. (2009). Cellular phenotypes of human model neurons (NT2) after differentiation in aggregate culture. Cell \& Tissue Research 336, 439-452.

33. Paquet-Durand, F. \& Bicker, G. (2007). Human model neurons in studies of brain cell damage and neural repair. Current Molecular Medicine 7, 541-554.

34. Podrygajlo, G., Song, Y., Schlesinger, F., Krampfl, K. \& Bicker, G. (2010). Synaptic currents and transmitter responses in human NT2 neurons differentiated in aggregate culture. Neuroscience Letters 468 , 207-210.

35. Ekong, T.A., Feavers, I.M. \& Sesardic, D. (1997). Recombinant SNAP-25 is an effective substrate for Clostridium botulinum type A toxin endopeptidase activity in vitro. Microbiology 143, 3337-3347.

36. Sesardic, D., Jones, R.G., Leung, T., Alsop, T. \& Tierney, R. (2004). Detection of antibodies against botulinum toxins. Movement Disorders: Official Journal of the Movement Disorder Society 19, Suppl. 8, S85-S91.

37. Gaines Das, R.E., Heath, A.B., Martin, H. \& 
Sesardic, D. (1999). Validation of in vitro assays for botulinum toxin: a case study. Developments in Biological Standardization 101, 267-276.

38. Jones, R.G.A., Ochiai, M., Liu, Y., Ekong, T. \&
Sesardic, D. (2008). Development of improved SNAP25 endopeptidase immune-assays for botulinum type A and $\mathrm{E}$ toxins. Journal of Immunological Methods 329, 92-101. 